\title{
ON POLYLOGARITHMS
}

\author{
by M. S. P. EASTHAM
}

(Received 31 May, 1963)

1. The $n$th order polylogarithm $L i_{n}(z)$ is defined for $|z| \leqq 1$ by

$$
L i_{n}(z)=\sum_{r=1}^{\infty} \frac{z^{r}}{r^{n}} \quad(n=2,3, \ldots)
$$

([4, p. 169], cf. $[2, \$ 1.11(14)$ and $\$ 1.11 .1])$. The definition can be extended to all values of $z$ in the $z$-plane cut along the real axis from 1 to $\infty$ by the formula

$$
L i_{n}(z)=\frac{z}{(n-1) !} \int_{0}^{\infty} \frac{t^{n-1}}{e^{t}-z} d t
$$

$[2, \S 1.11(3)]$. Then $L i_{n}(z)$ is regular in the cut plane, and there is a differential recurrence relation $[4$, p. 169$]$

$$
z L i_{n}^{\prime}(z)=L i_{n-1}(z) \quad(n \geqq 3) .
$$

It is convenient to extend the sequence $L i_{n}(z)$ backwards in the manner suggested by (2) and define

$$
L i_{1}(z)=z L i_{2}^{\prime}(z), \quad L i_{0}(z)=z L i_{1}^{\prime}(z), \ldots .
$$

Then $L i_{1}(z)=-\log (1-z)$, and $L i_{n}(z)$ is a rational function of $z$ for $n=0,-1,-2, \ldots$ Formula (2) now holds for all integers $n$.

2. We now prove

THEOREM. There is no pure recurrence relation of the form

$$
A_{0}(z) L i_{m}(z)+A_{1}(z) L i_{m-1}(z)+\ldots+A_{r}(z) L i_{m-r}(z)=0,
$$

where the $A_{n}(z)$ are algebraic functions of $z, A_{0}(z)$ is not identically zero, $m \geqq 1$, and $r \geqq m$ is allowed.

Suppose that there is a relation (3). Divide by $A_{0}(z)$, differentiate with respect to $z$, and use (2) for each $L i_{n}^{\prime}(z)$. We obtain an equation of the form

$$
B_{0}(z) L i_{m-1}(z)+\ldots+B_{r}(z) L i_{m-r-1}(z)=0,
$$

where $B_{0}(z)=\left\{A_{1}(z) / A_{0}(z)\right\}^{\prime}+1 / z$. Since the $A_{n}(z)$ are algebraic functions of $z$, so are the $B_{n}(z)$, and $B_{0}(z)$ is not identically zero. We now repeat the process until we obtain

$$
K_{0}(z) L i_{1}(z)+\ldots+K_{r}(z) L i_{-r+1}(z)=0,
$$

say, where the $K_{n}(z)$ are algebraic, and $K_{0}(z)$ is not identically zero. But (4) implies that $\log (1-z)$ is an algebraic function of $z$, which is a contradiction. Hence there is no relation of the form (3). 
3. A generating function. From (1) we have

$$
\sum_{n=2}^{\infty} w^{n-1} L i_{n}(z)=z \sum_{n=2}^{\infty} \frac{w^{n-1}}{(n-1) !} \int_{0}^{\infty} \frac{t^{n-1}}{e^{t}-z} d t=z \int_{0}^{\infty} \frac{e^{w t}-1}{e^{t}-z} d t,
$$

on inverting the order of integration and summation, this being justified by absolute convergence if $|w|<1$. The function on the right of (5) is thus a generating function for the $L i_{n}(z)(n \geqq 2)$.

A special case of (5) is the known formula $[2, \$ 1.17(5)]$

$$
\sum_{n=2}^{\infty} w^{n-1} \zeta(n)=-\psi(1-w)-\gamma \quad(|w|<1)
$$

where $\psi(x)$ is the logarithmic derivative of the gamma function $\Gamma(x)$ and $\gamma$ is Euler's constant. This is obtained from (5) on taking $z=1$ and using the formula [2, $\$ 1.7 .2(14)]$

$$
\psi(1+w)+\gamma=-\int_{0}^{\infty} \frac{e^{-w t}-1}{e^{t}-1} d t
$$

and the fact that $L i_{n}(1)=\zeta(n)$.

Next, let

$$
\sigma(n)=\sum_{r=1}^{\infty} \frac{(-1)^{r-1}}{r^{n}} .
$$

Then, since $\sigma(n)=\left(1-2^{-n+1}\right) \zeta(n),(6)$ gives

$$
\sum_{n=2}^{\infty} w^{n-1} \sigma(n)=\psi\left(1-\frac{1}{2} w\right)-\psi(1-w)
$$

This equation was used in [1] to evaluate the log-sine integrals

$$
\int_{0}^{\frac{1}{2} \pi}\{\log (2 \sin \theta)\}^{n} d \theta
$$

which also have other connexions with polylogarithms [4, pp. 148, 151-152, 184-185, 195-198].

4. Summation of series. By means of (5) we can sum various series involving polylogarithms. When $w$ is rational, say $w=p / q$, where $p$ and $q$ are integers, $q>0$, and $|p|<q$, the substitution $u=e^{-t / q}$ reduces the integral in (5) to the integral of a rational function of $u$, which can be evaluated. For example, when $w= \pm \frac{1}{2}$ and $z$ is real, we obtain the formulae

$$
\sum_{n=2}^{\infty} \frac{1}{2^{n-1}} L i_{n}(z)= \begin{cases}-2(-z)^{\frac{1}{2}} \tan ^{-1}(-z)^{\frac{1}{2}}+\log (1-z) & (z \leqq 0), \\ 2 z^{\frac{1}{2}} \log \left(1+z^{\frac{1}{2}}\right)+\left(1-z^{\frac{1}{2}}\right) \log (1-z) & (0 \leqq z<1), \\ 2 \log 2 & (z=1),\end{cases}
$$




$$
\sum_{n=2}^{\infty} \frac{(-1)^{n-1}}{2^{n-1}} L i_{n}(z)= \begin{cases}2(-z)^{-\frac{1}{2}} \tan ^{-1}(-z)^{\frac{1}{2}}+\log (1-z)-2 & (z<0), \\ 2 z^{-\frac{1}{2}} \log \left(1+z^{\frac{1}{2}}\right)+\left(1-z^{-\frac{1}{2}}\right) \log (1-z)-2 & (0<z<1) \\ 2 \log 2-2 & (z=1) .\end{cases}
$$

Formulae equivalent to (7) and (8) are given in [4, pp. 234-235] (see also [3]).

\section{REFERENCES}

1. F. Bowman, Note on the integral $\int_{0}^{\frac{1}{2} \pi}(\log \sin \theta)^{n} d \theta$, J. London Math. Soc. 22 (1947), 172-173.

2. A. Erdélyi, et al., Higher transcendental functions, Vol. I (New York, 1953).

3. J. W. L. Glaisher, Relations connecting sums of powers ..., Quart. J. Math.44 (1913), 170-186.

4. L. Lewin, Dilogarithms and associated functions (London, 1958).

ThE UNIVERSITY

READING 